\title{
MODAL BOOLEAN CONNEXIVE LOGICS: SEMANTICS AND TABLEAU APPROACH
}

\begin{abstract}
In this paper we investigate Boolean connexive logics in a language with modal operators: $\square, \diamond$. In such logics, negation, conjunction, and disjunction behave in a classical, Boolean way. Only implication is non-classical. We construct these logics by mixing relating semantics with possible worlds. This way, we obtain connexive counterparts of basic normal modal logics. However, most of their traditional axioms formulated in terms of modalities and implication do not hold anymore without additional constraints, since our implication is weaker than the material one. In the final section, we present a tableau approach to the discussed modal logics.

Keywords: Boolean connexive logics, connexive logic, modal Boolean connexive logics, modal logics, normal modal logics, possible worlds semantics, relatedness, relating logic, relating semantics, tableau methods.
\end{abstract}

\section{Introduction}

Aristotle's and Boethius' laws are of fundamental significance for the connexive logics. Negation and implication are the only connectives involved in them:

$$
\begin{gathered}
\sim(A \Rightarrow \sim A) \\
\sim(\sim A \Rightarrow A)
\end{gathered}
$$




$$
\begin{aligned}
& (A \Rightarrow B) \Rightarrow \sim(A \Rightarrow \sim B) \\
& (A \Rightarrow \sim B) \Rightarrow \sim(A \Rightarrow B)
\end{aligned}
$$

If we add any of these laws to the classical logic then by applying the modus ponens rule and substitution we obtain trivial, inconsistent logic - a set of all formulas. For this reason, in order to investigate connexive logics we need to interpret the negation or implication in a non-classical way. On the other hand, it is a natural idea to keep as close as possible to the classical logic while investigating the connexive logics by interpreting the Aristotelian and Boethian laws. This very idea guided us in our research published in paper [6]. There, we interpreted negation, conjunction, and disjunction in the classical Boolean way, leaving at the same time aside a broad spectrum of possible interpretations of implication by application of relating semantics to it. In the analyzed approach, the truth conditions for the implication consist not only of classical requirement that a antecedent is false or a consequent is true. There is an additional requirement that antecedent and consequent are interrelated by some binary relation $\mathrm{R}$.

In [6] we constructed 32 logics by determining respective classes of relations R. In two of them Aristotelian and Boethian laws hold and at the same time the negation, conjunction, and disjunction preserve the Boolean meaning. That is why we proposed the name Boolean connexive logics for such logics.

It is well known that a logical system solely based on the Aristotle's and Boethius' theses allows strange interpretations either of implication or of negation, which makes it far to weak for any reasonable applications. See [9] for historical review of this subject.

In the literature one could distinguish three approaches to the connexivity. The first one consists of proposals of specific logical systems designed for a given intended aims, such as [12], where connexive systems of conditionals are investigated. In the second approach, such as [7] and [8], some additional conditions are proposed to distinguish the well behaving connexive logics. One could also find the third approach, such as [10], where a comparison of different connexive logics is conducted. In the present paper we understand the connexive logic in a very general way, just as any set of sentences closed under substitution and modus ponens containing the Aristotle's and Boethian laws. This way, we investigate the structural properties of a broad spectrum of the sentential connexive logics. 
In a general way, the relating semantics was proposed in paper [4]. Its main notion - a relating relation - can be equipped with a large number of philosophical and not only philosophical motivations and interpretations. Two formulas can be related by $\mathrm{R}$ in many ways. For example, they could be related analytically, causally, thematically, temporally, etc. In this paper, the relating semantics is directly applied to the connexive implication. As a consequence, in this approach the connexive implication is true iff a antecedent is false or a consequent is true and simultaneously both are connected in some way. In the semantics, this connection is expressed by the relating relation.

In the present paper, we continue the investigations initiated in [6] by generalizing its results towards the area of modal logics. In a natural way, by modal Boolean connexive logics we mean a logic formulated in the sentential language with implication, classical negation, classical disjunction, classical conjunction, necessity and possibility operators satisfying the Aristotle's and Boethius' laws.

To express this in a short way: the modal Boolean connexive logic is a Boolean connexive logic defined in a modal language. The semantics considered here is a kind of combination of possible worlds semantics and relating semantics. As a consequence, we have two types of binary relations: a relating relation between formulas determining the meaning of implication and an accessibility relation on possible worlds defining modal operators. It appears that both kinds of relations affect each other and to some extent limit the traditional modal laws. A motivation for considering this particular combination of two semantics is natural. Since in [6] we considered the connexive logics without possible worlds, here we extend our ideas onto the possible worlds framework.

We define a number of connexive counterparts of the basic normal modal logics. However, most of their traditional axioms formulated in terms of modalities and implication do not hold anymore, since our implication is weaker than the material one. To make them valid we must impose some additional constraints on the relating semantics. In particular, we propose that modal operators have no influence on being related. We show the correspondence between both: relating as well as accessibility relations and modal axioms in all presented variants.

Additionally, as a decision procedure, in the last section we propose tableau methods for the connexive modal systems that constructed here. 


\section{Relating logics: syntax and semantics}

Let us consider the set of formulas For ${ }_{\mathbf{C P L}}$ of Classical Propositional Logic $(\mathrm{CPL})$, made up in a standard manner from: variables $\operatorname{Var}=\left\{p, q, r, p_{1}\right.$, $\left.q_{1}, r_{1}, \ldots\right\}$, one unary connective: $\neg$, four binary connectives: $\wedge, \vee, \rightarrow, \leftrightarrow$, and brackets: $),\left(\right.$. Let $\models_{\text {CPL }}$ be a consequence relation of CPL defined on For $_{\text {CPL }}$ by the set of all classical valuations of formulas from For ${ }_{\text {CPL }}$.

Whereas the set of formulas of Relating Logic (RL) For $\mathbf{R L}_{\mathbf{L}}$ is generated with $\vee$ ar, negation $\neg$, four binary connectives: $\wedge, \vee, \rightarrow, \leftrightarrow$ and four binary relating connectives that are relating counterparts of classical connectives: $\wedge^{\mathrm{w}}, \vee^{\mathrm{w}}, \rightarrow^{\mathrm{w}}, \leftrightarrow^{\mathrm{w}}$, and brackets: ), (. Thus, For ${ }_{\mathbf{C P L}} \subset$ For $_{\mathbf{R L}}$.

A model for the relating formulas is pair $\langle v, \mathrm{R}\rangle$, where $v$ : Var $\mapsto\{0,1\}$ and $\mathbf{R} \subseteq$ For $_{\mathbf{R L}} \times$ For $_{\mathbf{R L}}$. Function $v$ to any variable assigns either truth or falsity. Relation $\mathrm{R}$ is called relating relation. We have the following, general truth conditions for the relating formulas:

$$
\begin{aligned}
& \langle v, \mathrm{R}\rangle \models A \text { iff } v(A)=1, \quad \text { if } A \in \mathrm{Var} \\
& \langle v, \mathrm{R}\rangle \models \neg A \text { iff }\langle v, \mathrm{R}\rangle \not \models A \\
& \langle v, \mathrm{R}\rangle \models A \wedge^{\mathrm{w}} B \text { iff }\langle v, \mathrm{R}\rangle \models A \&\langle v, \mathrm{R}\rangle \models B \& \mathrm{R}(A, B) \\
& \langle v, \mathrm{R}\rangle \models A \vee^{\mathrm{w}} B \text { iff }[\langle v, \mathrm{R}\rangle \models A \text { or }\langle v, \mathrm{R}\rangle \models B] \& \mathrm{R}(A, B) \\
& \langle v, \mathrm{R}\rangle \models A \rightarrow^{\mathrm{w}} B \text { iff }[\langle v, \mathrm{R}\rangle \not A \text { or }\langle v, \mathrm{R}\rangle \models B] \& \mathrm{R}(A, B) \\
& \langle v, \mathrm{R}\rangle \models A \leftrightarrow^{\mathrm{w}} B \text { iff }[\langle v, \mathrm{R}\rangle \models A \text { iff }\langle v, \mathrm{R}\rangle \models B] \& \mathrm{R}(A, B) .
\end{aligned}
$$

As we can see, the relating connectives have intensional, and even hyperintensional character, since the Boolean conditions are not sufficient.

The set of all models for $\mathbf{R} \mathbf{L}$ will be denoted by $\mathbf{M}_{\mathbf{R} \mathbf{L}}$. By taking any subset $\mathbf{M}$ of $\mathbf{M}_{\mathbf{R L}}$ in the standard way, we define relating $\operatorname{logic} \models_{\mathbf{M}}$ :

$$
X \models_{\mathbf{M}} A \text { iff for all } \mathfrak{M} \in \mathbf{M} \text {, if } \mathfrak{M} \models X \text {, then } \mathfrak{M} \models A \text {. }
$$

The smallest relating logic is defined modulo all models. It is called RF in [4].

The first relating logic was probably proposed in [1], [2], [11]. However, in those studies, the authors analyzed a special kind of relating logics, called relatedness logic. There was considered a specific relation needed to define an extraordinary kind of content-related implication. On the other hand, the approach to the relating logics initiated in [4] is more general. We find 
the relatedness logic as a part of a much wider class of the relating logics with the multi-domain of applications. In principle, we could apply the relating semantics to any logic. Considering the formal conditions defining the classes of relating relations, one could determine the subclasses of $\mathbf{M}_{\mathbf{R L}}$, and in consequence, define a multitude of specific relating logics.

However, if we only take account of the relating binary part of RL formulas, meaning the smallest subset of For $\mathbf{R L}_{\mathbf{L}}$ closed under $\operatorname{Var}, \neg, \wedge^{\mathrm{w}}$, $\vee^{\mathrm{w}}, \rightarrow^{\mathrm{w}}, \leftrightarrow^{\mathrm{w}}$, and brackets ), (, we shall get set For $\mathrm{RL}_{\mathrm{w}}^{\mathrm{w}} \subset$ For $_{\mathbf{R L}}$ that

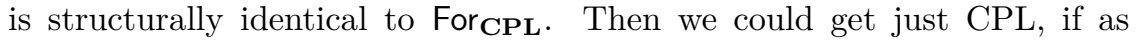
models we assumed all models $\langle v, \mathrm{R}\rangle$, where $\mathrm{R}$ is a universal relation, so $R=\operatorname{For}_{\mathbf{R} \mathbf{L}}^{\mathrm{w}} \times \operatorname{For}_{\mathbf{R} \mathbf{L}}^{\mathrm{w}}$.

In order to simplify the notation, we define the language of Boolean connexive logics as identical to For ${ }_{\mathbf{C P L}}$. Although in [6] we used the language generated with: variables $\operatorname{Var}=\left\{p, q, r, p_{1}, q_{1}, r_{1}, \ldots\right\}$; one unary connective: $\neg$; three binary connectives: $\wedge, \vee, \rightarrow^{\mathrm{w}}$ and brackets ), (. Also for the sake of simplicity, here instead of symbol $\rightarrow^{\text {w }}$ we shall use symbol $\rightarrow$. The obtained set, will be denoted by For ${ }_{\mathbf{C F}}$ (connexive formulas), so in fact For $_{\mathbf{C F}}=$ For $_{\mathbf{C P L}}$.

Now, implication $\rightarrow$ is intended to behave like a relating connective, while the other ones hold the classical, Boolean meaning.

Basic semantics for the Boolean connexive logics can be defined by the following truth conditions. Extensional for the Boolean operators:

$$
\begin{array}{ll}
\langle v, \mathrm{R}\rangle \models A \text { iff } v(A)=1, & \text { if } A \in \operatorname{Var} \\
\langle v, \mathrm{R}\rangle \models \neg A \text { iff }\langle v, \mathrm{R}\rangle \not A & \\
\langle v, \mathrm{R}\rangle \models A \wedge B \text { iff }\langle v, \mathrm{R}\rangle \models A \&\langle v, \mathrm{R}\rangle \models B & \\
\langle v, \mathrm{R}\rangle \models A \vee B \text { iff }\langle v, \mathrm{R}\rangle \models A \text { or }\langle v, \mathrm{R}\rangle \models B &
\end{array}
$$

and the intensional condition for relating implication $\rightarrow$ :

$$
\langle v, \mathrm{R}\rangle \models A \rightarrow B \text { iff }[\langle v, \mathrm{R}\rangle \not \models A \text { or }\langle v, \mathrm{R}\rangle \models B] \& \mathrm{R}(A, B) .
$$

Similarly, as in the modal logic, we treat $\mathrm{R}$ as a structure of given model. So, we assume: $\mathrm{R} \models A$ iff for all valuations of letters $v,\langle v, \mathrm{R}\rangle \models A$. Obviously, in order to accomodate the specific connexive laws, we had to distinguish some class of models. 


\section{Quasi-connexive and connexive Boolean logics}

Before we propose some extension of Boolean connexive logic to the modal language, let us recapitulate the basic facts from [6].

Let For $_{\mathbf{C F}}^{2}$ denote For $_{\mathbf{C F}} \times$ For $_{\mathbf{C F}}$. Let $\mathrm{R} \subseteq$ For $_{\mathbf{C F}}^{2}$. To define suitable classes of models for the Boolean connexive logics, we require the complement of relating relation R. $A \tilde{\mathrm{R}} B$ means that the relation $A \mathrm{R} B$ does not hold.

We define some classes of relations $\mathrm{R}$ determined by the following conditions:

(a1) $\mathrm{R}$ is (a1) iff for all $A \in$ For $_{\mathrm{CF}}, A \widetilde{\mathrm{R}} \neg A$

(a2) $\mathrm{R}$ is (a2) iff for all $A \in$ For $_{\mathbf{C F}}, \neg A \widetilde{\mathrm{R}} A$

(b1) $\mathrm{R}$ is (b1) iff for all $A, B \in$ For $_{\mathbf{C F}}$ :

- if $A \mathrm{R} B$, then $A \widetilde{\mathrm{R}} \neg B$

- $(A \rightarrow B) \mathrm{R} \neg(A \rightarrow \neg B)$

(b2) $\mathrm{R}$ is (b2) iff for all $A, B \in$ For $_{\mathbf{C F}}$ :

- if $A \mathrm{R} B$, then $A \widetilde{\mathrm{R}} \neg B$

- $(A \rightarrow \neg B) \mathrm{R} \neg(A \rightarrow B)$

(c1) $\mathrm{R}$ is (c1) iff for all $A, B \in$ For $_{\mathbf{C F}}$, if $A \mathrm{R} B$ then $\neg A \mathrm{R} \neg A$.

If $\mathrm{R}$ is (c1), it is often called closed under negation.

In paper [6] we showed that conditions (a1), (a2), (b1), (b2), (c1) were independent. Moreover, we proved the following theorem:

ThEOREM 3.1 (Correspondence theorem). Let $R \subseteq$ For $_{C F}^{2}$ satisfy (c1). Then:

$$
\begin{aligned}
& R \text { is (a1) } \Leftrightarrow R \models \neg(A \rightarrow \neg A) \\
& R \text { is (a2) } \Leftrightarrow R \models \neg(\neg A \rightarrow A) \\
& R \text { is (b1) } \Leftrightarrow R \models(A \rightarrow B) \rightarrow \neg(A \rightarrow \neg B) \\
& R \text { is (b2) } \Leftrightarrow R \models(A \rightarrow \neg B) \rightarrow \neg(A \rightarrow B) .
\end{aligned}
$$

Condition (c1) is needed there only for proving the inference from the left to right-hand side part of each condition, hence we have the following: THEOREM 3.2. Suppose that $R \subseteq$ For $_{C \boldsymbol{F}}^{2}$ :

(1) $R$ is (a1) $\Rightarrow R \models \neg(A \rightarrow \neg A)$

(2) $R$ is (a2) $\Rightarrow R \models \neg(\neg A \rightarrow A)$ 
(3) $R$ is (b1) $\Rightarrow R \models(A \rightarrow B) \rightarrow \neg(A \rightarrow \neg B)$

(4) $R$ is (b2) $\Rightarrow R \models(A \rightarrow \neg B) \rightarrow \neg(A \rightarrow B)$.

The theorems above allow us to construct a dozen of logical systems by imposing some limitations on the relating models. Some of them are Boolean connexive logics, other are not. Since the conditions from set $\{(\mathrm{a} 1),(\mathrm{a} 2),(\mathrm{b} 1),(\mathrm{b} 2),(\mathrm{c} 1)\}$ are independent, then any two of its subsets determine different logical systems. Since there are $2^{5}=32$ subsets, so the combinations of conditions determine 32 different logical systems. Among them, two logics are Boolean connexive logics: determined by (a1), (a2), (b1), (b2) in one case, determined by (a1), (a2), (b1), (b2), (c1) in the other. The logic determined by conditions (a1), (a2), (b1), (b2) is the least Boolean connexive logic.

Let us assume that by Boolean quasi-connexive logic we mean a logic determined by set of all models satisfying at least one, but not all of conditions: (a1), (a2), (b1), (b2). Then among 32 logics determined by the above models: (i) two are neither connexive, nor quasi-connexive - zero of Aristotelian or Boethian conditions are satisfied; (ii) 28 logics are quasiconnexive - at least one, but not all conditions are satisfied.

\section{Emerging modal Boolean connexive logics}

Now we extend the language For $_{\mathbf{C F}}$ by closing it additionally under two unary modal operators $\square$ and $\diamond$. The language constructed this way will be denoted by For CMF (connexive modal formulas).

By a model for For CMF we mean quadruple $\left\langle W, Q,\left\{\mathrm{R}_{w}\right\}_{w \in W}, v\right\rangle$ where:

- $W$ is a non empty set of "possible worlds"

- $Q \subseteq W \times W$ is an accessibility relation

- $\left\{\mathrm{R}_{w}\right\}_{w \in W}$ is a family of relations that contsists of $\mathrm{R}_{w} \subseteq$ For $_{\mathrm{CMF}} \times$ For ${ }_{\mathrm{CMF}}$, for any $w \in W$, so a particular $\mathrm{R}_{w}$ is a relating relation, for any possible world $w \in W$

- $v: W \times \operatorname{Var} \longrightarrow\{0,1\} v$ is a valuation of sentential letters in worlds.

Let us note again that a model contains two types of binary relations. Relation $Q$ is a standard accessibility relation between possible words, while indexed $\mathrm{R}$ is a binary relation between formulas, one for each possible world $w$ in $W$. 
For any model $\mathfrak{M}=\left\langle W, Q,\left\{\mathrm{R}_{w}\right\}_{w \in W}, v\right\rangle$ and any $w \in W$, we define a satisfaction relation in the following way:

$$
\begin{array}{ll}
\mathfrak{M}, w \models A \text { iff } v(w, A)=1, & \text { if } A \in \operatorname{Var} \\
\mathfrak{M}, w \models \neg A \text { iff } \mathfrak{M}, w \not \models A & \\
\mathfrak{M}, w \models A \wedge B \text { iff } \mathfrak{M}, w \models A \& \mathfrak{M}, w \models B & \\
\mathfrak{M}, w \models A \vee B \text { iff } \mathfrak{M}, w \models A \text { or } \mathfrak{M}, w \models B & \\
\mathfrak{M}, w \models \square A \text { iff } \forall_{u \in W}(w Q u \Rightarrow \mathfrak{M}, u \models A) & \\
\mathfrak{M}, w \models \diamond A \text { iff } \exists_{u \in W}(w Q u \& \mathfrak{M}, u \models A) & \\
\mathfrak{M}, w \models A \rightarrow B \text { iff }[\mathfrak{M}, w \not A \text { or } \mathfrak{M}, w \models B] \& A \mathrm{R}_{w} B . &
\end{array}
$$

Let $\mathfrak{M}=\left\langle W, Q,\left\{\mathrm{R}_{w}\right\}_{w \in W}, v\right\rangle$. We will say that formula $A$ is true in model $\mathfrak{M}$ (in symbols: $\mathfrak{M} \models A$ ) iff for any possible world $w$ in $W: \mathfrak{M}, w \models A$. If $X \subseteq$ For $_{\mathbf{C M F}}$ and $w \in W$, then we say that $\mathfrak{M}, w \models X$ iff for all $A \in X: \mathfrak{M}, w \models A$.

Given class of models $\mathrm{C}$ and $X \cup\{A\} \subseteq$ For $_{\mathbf{C F}}$, we will say that $X$ entails $A$ modulo $\mathrm{C}$ (in symbols $X \models_{\mathrm{C}} A$ ) iff for all $\mathfrak{M}=\left\langle W, Q,\left\{\mathrm{R}_{w}\right\}_{w \in W}, v\right\rangle \in \mathrm{C}$ and all $w \in W$ : if $\mathfrak{M}, w \models X$, then $\mathfrak{M}, w \models A$. Clearly, $A$ is a tautology of $\mathrm{C}$ iff $\emptyset \models_{\mathrm{C}} A$ (in short: $\models_{\mathrm{C}} A$ ), where $\emptyset$ is an empty set. Traditionally, we say that model $\mathfrak{M}=\left\langle W, Q,\left\{\mathrm{R}_{w}\right\}_{w \in W}, v\right\rangle$ is based on $\langle W, Q\rangle$.

Now, we do not deal with a single relation, but with a family of relations. So, the relating structure is $\left\langle W,\left\{R_{w}\right\}_{w \in W}\right\rangle$, instead of a single $\mathrm{R}$. But, since it is clear that set $W$ indexes a set of relations, therefore it can be reduced to $\left\{R_{w}\right\}_{w \in W}=\left\{R_{w}: w \in W\right\}$, for some $W$.

Taking a modal frame $\langle W, Q\rangle$, we can mix it with relating structures, and vice versa. However, if we do not impose any constraints on accessibility relation $Q$, we can talk only of a family of relating relations. Consequently, we assume a definition of being true in the mixed structure and separately in the relating structure:

$\left\langle W, Q,\left\{R_{w}\right\}_{w \in W}\right\rangle \models A$ iff $\left\langle W, Q,\left\{\mathrm{R}_{w}\right\}_{w \in W}, v\right\rangle \models A$, for all valuations of letters $v$ in $W$

$\left\{R_{w}\right\}_{w \in W} \models A$ iff $\left\langle W, Q,\left\{\mathrm{R}_{w}\right\}_{w \in W}, v\right\rangle \models A$, for all accessibility relations $Q \in W \times W$ of some kind (in some class of accessibility relations) and all valuations of letters $v$ in $W$. 
Below, we define some properties of relation $\mathrm{R}$ corresponding to the Aristotle's and Boethius' theses. They are natural modal counterparts of conditions (a1), (a2), (b1), (b2), (c1) from [6], extended to each family of relations indexed by worlds from some $W$. So, let $\left\{R_{w}\right\}_{w \in W}$ be a family of relating relations indexed by worlds from some $W$.

(Ma1) $\left\{\mathrm{R}_{w}\right\}_{w \in W}$ is (Ma1) iff for all $A \in$ For $_{\mathrm{CMF}}, \forall_{w \in W} A \widetilde{\mathrm{R}_{w}} \neg A$

(Ma2) $\left\{\mathrm{R}_{w}\right\}_{w \in W}$ is (Ma2) iff for all $A \in$ For $_{\text {CMF }}, \forall_{w \in W} \neg A \widetilde{\mathrm{R}_{w}} A$

(Mb1) $\left\{\mathrm{R}_{w}\right\}_{w \in W}$ is (Mb1) iff for all $A, B \in$ For $_{\mathbf{C M F}}, \forall_{w \in W}$ :

- if $A \mathrm{R}_{w} B$, then $A \widetilde{\mathrm{R}_{w}} \neg B$

- $(A \rightarrow B) \mathrm{R}_{w} \neg(A \rightarrow \neg B)$

(Mb2) $\left\{\mathrm{R}_{w}\right\}_{w \in W}$ is (Mb2) iff for all $A, B \in$ For $_{\mathbf{C M F}}, \forall_{w \in W}$ :

- if $A \mathrm{R}_{w} B$, then $A \widetilde{\mathrm{R}_{w}} \neg B$

- $(A \rightarrow \neg B) \mathrm{R}_{w} \neg(A \rightarrow B)$

(Mc1) $\left\{\mathrm{R}_{w}\right\}_{w \in W}$ is (Mc1) iff for all $A, B \in$ For $_{\mathbf{C M F}}, \forall_{w \in W}\left(A \mathrm{R}_{w} B \Rightarrow\right.$ $\left.\neg A \mathrm{R}_{w} \neg B\right)$.

Clearly, if we say that a model satisfies one (or more) of the above conditions, we mean that in fact its family of relating relations does it, and the model inherits this property. For example, model $\left\langle W, Q,\left\{R_{w}\right\}_{w \in W}, v\right\rangle$ is (Mb2) iff $\left\{R_{w}\right\}_{w \in W}$ is (Mb2) etc. Let us assume that the remark applies to all properties we introduce in the paper here and further.

We have a similar theorem to the Correspondence Theorem in [6]. This time, it is extended to the modal context.

Theorem 4.1 (Modal Correspondence Theorem). Let $\left\{R_{w}\right\}_{w \in W}$ be a family of relating relations for some $W$.

If $\left\{R_{w}\right\}_{w \in W}$ is (Mc1), then:

$$
\begin{aligned}
& \left\{R_{w}\right\}_{w \in W} \text { is (Ma1) iff }\left\{R_{w}\right\}_{w \in W} \models \neg(A \rightarrow \neg A) \\
& \left\{R_{w}\right\}_{w \in W} \text { is (Ma2) iff }\left\{R_{w}\right\}_{w \in W} \models \neg(\neg A \rightarrow A) \\
& \left\{R_{w}\right\}_{w \in W} \text { is (Mb1) iff }\left\{R_{w}\right\}_{w \in W} \models(A \rightarrow B) \rightarrow \neg(A \rightarrow \neg B) \\
& \left\{R_{w}\right\}_{w \in W} \text { is (Mb2) iff }\left\{R_{w}\right\}_{w \in W} \models(A \rightarrow \neg B) \rightarrow \neg(A \rightarrow B) .
\end{aligned}
$$

Proof: The proof is similar to the proof of theorem 6.1 in [6], where we proved the Correspondence Theorem for conditions (a1), (a2), (b1), 
(b2), (c1). In fact, we show the equivalences for all relations belonging to $\left\{\mathrm{R}_{w}\right\}_{w \in W}$ in the identical ways as we did in [6].

Similarly as in the non-modal case, condition (Mc1) is necessary only to prove the inference from the left to the right part of each condition. Hence, by definitions of the conditions we have:

THEOREM 4.2. Let $\left\{R_{w}\right\}_{w \in W}$ be a family of relating relations for some set $W$. Then:

$$
\begin{aligned}
& \left\{R_{w}\right\}_{w \in W} \text { is (Ma1) } \Rightarrow\left\{R_{w}\right\}_{w \in W} \models \neg(A \rightarrow \neg A) \\
& \left\{R_{w}\right\}_{w \in W} \text { is (Ma2) } \Rightarrow\left\{R_{w}\right\}_{w \in W} \models \neg(\neg A \rightarrow A) \\
& \left\{R_{w}\right\}_{w \in W} \text { is (Mb1) } \Rightarrow\left\{R_{w}\right\}_{w \in W} \models(A \rightarrow B) \rightarrow \neg(A \rightarrow \neg B) \\
& \left\{R_{w}\right\}_{w \in W} \text { is (Mb2) } \Rightarrow\left\{R_{w}\right\}_{w \in W} \models(A \rightarrow \neg B) \rightarrow \neg(A \rightarrow B) .
\end{aligned}
$$

Here, we can repeat the maneuver we did in [6]. So, first we have some theorem on the independence of modal versions of the connexive conditions.

ThEOREM 4.3. The conditions (Ma1), (Ma2), (Mb1), (Mb2), (Mc1) are independent.

Proof: Since non-modal conditions (a1), (a2), (b1), (b2), (c1) are independent (theorem 6.2 in [6]), so (Ma1), (Ma2), (Mb1), (Mb2), (Mc1) are also independent, which is established by singletons $W=\{w\}$ and $\left\{R_{w}\right\}$. However, if a model requires more worlds (for example, because of some special requirements put on the accessibility relation $Q$ ), then since relating relations included in one model are independent from each other, so we can extend the singleton models to models with a bigger cardinality, properly.

Secondly, similarly as in the non-modal case, since the conditions (Ma1), (Ma2), (Mb1), (Mb2), (Mc1) are independent, then each subset of set $\{(\mathrm{Ma} 1),(\mathrm{Ma} 2),(\mathrm{Mb} 1),(\mathrm{Mb} 2),(\mathrm{Mc} 1)\}$ determines a different family of relations $\left\{\mathrm{R}_{w}\right\}_{w \in W}$, i.e. we have $2^{5}=32$ of families of such kind. Each of them determines a different logical system.

Among these 32 logics determined by these models: one is neither connexive, nor quasi-connexive; 29 logics are quasi-connexive - at least one, but not all connexive laws are valid; but two logics are really connexive - their models satisfy conditions (Ma1), (Ma2), (Mb1), (Mb2) and possibly also (Mc1). The logic determined only by conditions (Ma1), (Ma2), 
(Mb1), (Mb2) is the least modal Boolean connexive logic. Surely, the top of this lattice is the inconsistent logic defined on For ${ }_{\mathbf{C F}}$ by an empty set of models. Let us note that these are basic variants, since by imposing some requirements on accessibility relation $Q$ we probably multiply the number of logics.

\section{Modal aspect of the Boolean connexive logics}

The very interesting question is whether our modal Boolean connexive logics are normal as modal logics. It is widely accepted that a modal logic is normal iff it is closed under necessitation rule: $\models A \Rightarrow \models \square A$ and contains axiom $\mathrm{K}: \square(A \rightarrow B) \rightarrow(\square A \rightarrow \square B)$. However, since in the language we have classical negation $\neg$ and diamond $\diamond$, hence also the so called dual should be satisfied: $\neg \diamond \neg A \rightarrow \square A$ and $\square A \rightarrow \neg \diamond \neg A$ (we do not dispose of the equivalence in the language, then the dual must be expressed as two opposite implications). As we know, our relating implication is much weaker than the material one. This is the reason why our modal Boolean connexive logics are not normal in the given sense. Also, if we limit the class of models by imposing specific conditions on accessibility relation $Q$ (imposing reflexivity, transitivity or symmetry etc.), we do not get axioms that are characteristic for the extensions of the normal modal logic, expressed as the appropriate implications. The next claim states this.

Claim 5.1. If $C$ is a class of all models that satisfy a subset of set of conditions $\{(M a 1),(M a 2),(M b 1),(M b 2),(M c 1)\}$, then the following facts hold:

(a) $\models_{C} A \Rightarrow \models{ }_{C} \square A$

(b) $\forall \forall_{C} \square A \rightarrow \neg \diamond \neg A$

(c) $\forall \forall_{C} \neg \diamond \neg A \rightarrow \square A$

(d) $\forall \nvdash_{C} \square(A \rightarrow B) \rightarrow(\square A \rightarrow \square B)$

(e) $\forall \nvdash_{C} \square A \rightarrow \diamond A$

(f) $\forall{ }_{C} \square A \rightarrow A$

(g) $\forall \models_{C} \square A \rightarrow \square \square A$

(h) $\forall_{C} A \rightarrow \square \diamond A$

(i) $\forall{ }_{C} \diamond A \rightarrow \square \diamond A$
Necessitation rule holds

Dual does not hold

Dual does not hold axiom $\mathrm{K}$ does not hold axiom $D$ does not hold, even with serial $Q$ axiom $T$ does not hold, even with reflexive $Q$ axiom 4 does not hold, even with transitive $Q$ axiom $B$ does not hold, even with symmetrical $Q$ axiom 5 does not hold, even with Euclidean $Q$.

Proof: We take class of models $\mathrm{C}$, consequence relation $\models_{\mathrm{C}}$, and formulas $A, B \in$ For $_{\mathrm{CMF}}$. 
For (a) let us assume that $\models_{\mathrm{C}} A$. Given model $\mathfrak{M}=\langle W, Q$, $\left.\left\{\mathrm{R}_{w}\right\}_{w \in W}, v\right\rangle \in \mathrm{C}$ and world $w \in W$. $\mathfrak{M}, w \models \square A$ iff for all $u \in W$ : $w Q w^{\prime} \Rightarrow \mathfrak{M}, u \models A$. So we take any such $u \in W$ that $w Q u$. However, by the assumption, in all models $\mathfrak{M}^{\prime}=\left\langle W^{\prime}, Q^{\prime},\left\{\mathrm{R}_{w}\right\}_{w \in W^{\prime}}, v^{\prime}\right\rangle$ in $\mathrm{C}$, all worlds $w^{\prime}$ in $W^{\prime}: \mathfrak{M}^{\prime}, w^{\prime} \models A$. Therefore, $\mathfrak{M}^{\prime}, u \models A$, and by arbitrariness of model and world: $\models_{\mathrm{C}} \square A$.

For (b) we take model $\mathfrak{M}=\left\langle W, Q,\left\{\mathrm{R}_{w}\right\}_{w \in W}, v\right\rangle$, where for some world $w \in W: \square \widetilde{A \mathrm{R}_{w}} \neg \diamond \neg A$. By definition, class of models $\mathrm{C}$ consists of all models of some kind that does not exclude such models. In consequence, $\mathfrak{M}, w \not \forall \square A \rightarrow \neg \diamond \neg A$, so $\mathfrak{M} \not \forall \square A \rightarrow \neg \diamond \neg A$, and finally $\forall_{\mathrm{C}} \square A \rightarrow$ $\neg \diamond \neg A$.

For (d) $\nvdash_{\mathrm{C}} \square(A \rightarrow B) \rightarrow(\square A \rightarrow \square B)$, it is enough to take model $\mathfrak{M}=$ $\left\langle W, Q,\left\{\mathrm{R}_{w}\right\}_{w \in W}, v\right\rangle$, where for some world $w \in W: \square(A \rightarrow B) \widetilde{\mathrm{R}_{w}}(\square A \rightarrow$ $\square B)$, so axiom $\mathrm{K}$ does not hold.

For (i) $\forall_{\mathrm{C}} \diamond A \rightarrow \square \diamond A$, we take model $\mathfrak{M}=\left\langle W, Q,\left\{\mathrm{R}_{w}\right\}_{w \in W}, v\right\rangle$, where for some world $w \in W: \diamond A \widetilde{\mathrm{R}_{w}} \square \diamond A$. Since relation $Q$ is Euclidean, so $\mathfrak{M}, w \models_{\mathrm{C}} \diamond A$ implies $\mathfrak{M}, w \models_{\mathrm{C}} \square \diamond A$. However, $\nvdash_{\mathrm{C}} \diamond A \rightarrow \square \diamond A$, because $\diamond A \widetilde{\mathrm{R}_{w}} \square \diamond A$. Hence, axiom 5 does not hold, even with Euclidean $Q$.

The remaining cases we prove in a very similar way, by indicating counterexamples. They all base upon the fact that to falsify implication it is enough to find such a relating relation satisfying the conditions (Ma1), (Ma2), (Mb1), (Mb2), (Mc1) that the implication antecedent and the implication consequent do not relate.

Now, we would like to enhance our logics a bit, by making all of the mentioned formulas true. One of the possibilities is to impose more conditions. Sometimes they turn out to correspond with the appropriate formulas, but not always is this a case. Let us start with axiom $\mathrm{K}$. We can state the sufficient, but not necessary conditions for it. Beforehand, we assume some notation. We assume that if $\mathfrak{M}=\left\langle W, Q,\left\{\mathrm{R}_{w}\right\}_{w \in W}, v\right\rangle$ is a model for language For ${ }_{\mathrm{CMF}}$, then $W^{\mathfrak{M}}=W, Q^{\mathfrak{M}}=Q$.

Claim 5.2. Let $C$ be a class of all models such that for all $\mathfrak{M}=\langle W, Q$, $\left.\left\{R_{w}\right\}_{w \in W}, v\right\rangle \in C$, for all $w \in W$, for all $A, B \in$ For $_{C M F}$ :

$(\mathrm{K})$ :

(1) $\square(A \rightarrow B) R_{w}(\square A \rightarrow \square B)$

(2) $\forall_{u \in W}\left(w Q u \Rightarrow A R_{u} B\right) \Rightarrow \square A R_{w} \square B$. 
Then:

$$
\models_{C} \square(A \rightarrow B) \rightarrow(\square A \rightarrow \square B) .
$$

Proof: Given any model $\mathfrak{M} \in C$ satisfying (1) and (2), we will show that for any possible world $w \in W: \mathfrak{M}, w \models \square(A \rightarrow B) \rightarrow(\square A \rightarrow \square B)$. From (1) and definition of satisfaction relation it is enough to show that if $\mathfrak{M}, w \models \square(A \rightarrow B)$ then: a) $\square A \mathrm{R}_{w} \square B$ and b) if $\mathfrak{M}, w \models \square A$, then $\mathfrak{M}, w \models \square B$.

a) From $\mathfrak{M}, w \models \square(A \rightarrow B)$ we have then for all $u \in W$ such that $w Q u$ $\mathfrak{M}, u \models A \rightarrow B$, hence $A \mathrm{R}_{u} B$, then from (2) $\square A \mathrm{R}_{w} \square B$.

b) Suppose that $\mathfrak{M}, w \models \square(A \rightarrow B)$ and $\mathfrak{M}, w \models \square A$, then for any $u \in W$ such that $w Q u, \mathfrak{M}, u \models A \rightarrow B$ and $\mathfrak{M}, u \models A$, hence $\mathfrak{M}, w \models \square B$.

Obviously condition axiom $\mathrm{K}$ entails (1) however it does not entail (2). Let us take the following model $\mathfrak{M}=\left\langle W, Q,\left\{\mathrm{R}_{w}\right\}_{w \in W}, v\right\rangle$, where $W=$ $\{w\}, Q=\{\langle w, w\rangle\}$, and $R_{w}=\{\langle p \vee \neg p, p \wedge \neg p\rangle\} \cup\{\langle\square(A \rightarrow B), \square A \rightarrow$ $\square B\rangle: A, B \in$ For $\left._{\text {CMF }}\right\} \cup\left\{\langle\square A, \square B\rangle: A, B \in\right.$ For $_{\text {CMF }}$ and $A \neq p \vee \neg p$ or $B \neq p \wedge \neg p\}$, and $v(w, x)=1$, for all $x \in$ Var.

We can see that $\mathfrak{M}, w \models \square(A \rightarrow B) \rightarrow(\square A \rightarrow \square B)$, for all $A, B \in$ For ${ }_{\text {CMF. }}$. However, condition (2) is falsified because $\forall_{u \in W}(w Q u \Rightarrow p \vee$ $\left.\neg p \mathrm{R}_{u} p \wedge \neg p\right)$, but $\square(p \vee \neg p) \widetilde{\mathrm{R}}_{w} \square(p \wedge \neg p)$.

For the remaining formulas we have sufficient as well as necessary conditions. The notations on the right denote conditions for the appropriate axioms on the left.

Claim 5.3. Let $C$ be a class of models. The following conditions are fulfilled:

(Du1) $\quad \models_{C} \square A \rightarrow \neg \diamond \neg A$ iff $\forall_{\mathfrak{M} \in C} \forall_{w \in W^{\mathfrak{M}}} \square A R_{w} \neg \nabla \neg A$

(Du2) $\quad \models_{C} \neg \nabla \neg A \rightarrow \square A$ iff $\forall_{\mathfrak{M} \in C} \forall_{w \in W^{\mathfrak{M}}} \neg \diamond \neg A R_{w} \square A$

(D) if $C$ is based on serial frames, then:

$$
\models_{C} \square A \rightarrow \diamond A \text { iff } \forall_{\mathfrak{M} \in C} \forall_{w \in W^{\mathfrak{M}}} \square A R_{w} \diamond A
$$

(T) if $C$ is based on reflexive frames, then:

$$
\models_{C} \square A \rightarrow A \text { iff } \forall_{\mathfrak{M} \in C} \forall_{w \in W^{\mathfrak{M}}} \square A R_{w} A
$$


(4)

if $C$ is based on transitive frames, then:

$$
\models_{C} \square A \rightarrow \square \square A \text { iff } \forall_{\mathfrak{M} \in C} \forall_{w \in W^{\mathfrak{M}}} \square A R_{w} \square \square A
$$

(B) if $C$ is based on symmetrical frames, then:

$$
\models_{C} A \rightarrow \square \diamond A \text { iff } \forall_{\mathfrak{M} \in C} \forall_{w \in W \mathfrak{M}} A R_{w} \square \diamond A
$$

$$
\begin{aligned}
& \text { if } C \text { is based on Euclidean frames, then: } \\
& \qquad \models_{C} \diamond A \rightarrow \square \diamond A \text { iff } \forall_{\mathfrak{M} \in C} \forall_{w \in W^{\mathfrak{M}}} \diamond A R_{w} \square \diamond A .
\end{aligned}
$$

Proof: Please note that in each case above the right-hand side condition grants that the antecedent and the consequent of implication on the lefthand side relate with respect to the relating relation. It directly shows that each right-hand side is a necessary condition. To prove that each right-hand side condition is sufficient it is enough to note that each lefthand side condition is true if we replace $\rightarrow$ by teh material implication.

The conditions presented in the latter fact may seem to be rather natural syntactic conditions. It is the case - we believe - because we treat modalities very literally. However there exists another option, much less obvious that is formally reduced to the demodalization.

\section{Demodalization and double negation}

Any modality can be treated - due to the Latin etymology of the word "modality" - as the way a modalized proposition holds. Term modality comes from the Latin world modus which means a way; a way that something happens.

An option of non-treating modalities literally is to assume that modalities $\square, \diamond$ add nothing to the content of propositions modalized by them. For some people it may sound controversial, but modus means a way, not a content.

We begin by considering an example. Below, we have two non-modal propositions:

$p:=$ Nicolaus Copernicus was born in Toruń

$q:=$ Torun is in Poland. 
It is obvious that $p$ and $q$ are connected somehow (for example, by sharing the city):

$$
p \mathrm{R} q .
$$

The question may appear if when we add modalities to them, will they still be connected? Insisting on the option that modalities bring nothing to the content, but only change the way or the status of proposition, it does not seem strange that one can find sentences $\diamond p$ and $\square q$ still connected. Also the subsequent iterations should not change this situation, if $\diamond p \mathrm{R} \square q$, then by adding the successive modalities we get the pair of connected propositions: $\square \diamond p \mathrm{R} \square \square q$ etc. The inverse direction should be treated as intuitive, too. Why? Because if we think the modalities bring nothing to the content, then the two modalized propositions are connected through the fact their non-modal components are connected. If so, then we assume generally:

$$
p \mathrm{R} q \Longleftrightarrow \circ_{1}, \ldots, \circ_{n} p \mathrm{R} \bullet \bullet_{1}, \ldots, \bullet_{m} q
$$

where $1 \leq n, m$ and $\circ_{1}, \ldots, \circ_{n}, \bullet_{1}, \ldots, \bullet_{m} \in\{\square, \diamond\}$.

Since the initial, non-modal sentences can be more complex than only sentential letters, we introduce a special function that removes modalities from the structure of sentences. By demodalization we mean mapping $\mathrm{d}:$ For $_{\mathrm{CMF}} \longrightarrow$ For $_{\mathrm{CPL}}$, determined by conditions:

$$
\begin{array}{lr}
\mathrm{d}(A)=A & A \in \mathrm{Var} \\
\mathrm{d}(\neg A)=\neg \mathrm{d}(A) & * \in\{\wedge, \vee, \rightarrow\} \\
\mathrm{d}(A * B)=\mathrm{d}(A) * \mathrm{~d}(B) & \circ \in\{\square, \diamond\} . \\
\mathrm{d}(\circ A)=\mathrm{d}(A) &
\end{array}
$$

Claim 6.1. Let $C$ be a class of models such that for all $\mathfrak{M} \in C$ and for all $A, B \in$ For $_{C M F}$ :

$$
\forall_{w \in W^{\mathfrak{M}}}\left(A R_{w} B \Longleftrightarrow d(A) R_{w} d(B)\right)
$$

\footnotetext{
${ }^{1}$ It is worth to mention that in [5] we introduced a very similar demodalization function. It was in the context of deontic logic while our aim was to underline and preserve the deontic relationships between the sentences related to different changing possible deontic worlds. We assumed that such relationship did not depend on the modal status, but on what a content of sentences was.
} 
Then the following conditions are fulfilled:

$$
\begin{aligned}
& (K+(d)) \quad \models_{C} \square(A \rightarrow B) \rightarrow(\square A \rightarrow \square B) \text { iff } \\
& \forall_{\mathfrak{M} \in C} \forall_{w \in W^{\mathfrak{M}}} d(A \rightarrow B) R_{w} d(A \rightarrow B) \\
& (D u 1+(d)) \quad \models_{C} \square A \rightarrow \neg \diamond \neg A \text { iff } \forall_{\mathfrak{M} \in C} \forall_{w \in W^{\mathfrak{M}}} d(A) R_{w} d(\neg \neg A) \\
& (D u 2+(d)) \quad \models{ }_{C} \neg \nabla \neg A \rightarrow \square A \text { iff } \forall_{\mathfrak{M} \in C} \forall_{w \in W^{\mathfrak{M}}} d(\neg \neg A) R_{w} d(A) \\
& (D+(d)) \quad \text { if } C \text { is based on serial frames, then: } \\
& \models_{C} \square A \rightarrow \diamond A \text { iff } \forall_{\mathfrak{M} \in C} \forall_{w \in W^{\mathfrak{M}}} d(A) R_{w} d(A) \\
& (T+(d)) \quad \text { if } C \text { is based on reflexive frames, then: } \\
& \models_{C} \square A \rightarrow A \text { iff } \forall_{\mathfrak{M} \in C} \forall_{w \in W^{\mathfrak{M}}} d(A) R_{w} d(A) \\
& (4+(d)) \quad \text { if } C \text { is based on transitive frames, then: } \\
& \models_{C} \square A \rightarrow \square \square A \text { iff } \forall_{\mathfrak{M} \in C} \forall_{w \in W^{\mathfrak{M}}} d(A) R_{w} d(A) \\
& (B+(d)) \quad \text { if } C \text { is based on symmetrical frames, then: } \\
& \models_{C} A \rightarrow \square \diamond A \text { iff } \forall_{\mathfrak{M} \in C} \forall_{w \in W^{\mathfrak{M}}} d(A) R_{w} d(A) \\
& \text { (5+(d)) if } C \text { is based on Euclidean frames, then: } \\
& \models_{C} \diamond A \rightarrow \square \diamond A \text { iff } \forall_{\mathfrak{M} \in C} \forall_{w \in W^{\mathfrak{M}}} d(A) R_{w} d(A) .
\end{aligned}
$$

Proof: Let us assume a class of all models $\mathrm{C}$ that all satisfy a subset of set of conditions $\{(\mathrm{Ma} 1),(\mathrm{Ma} 2),(\mathrm{Mb} 1),(\mathrm{Mb} 2),(\mathrm{Mc} 1)\}$, and additionally let all $\mathfrak{M} \in \mathrm{C}$ satisfy condition (d).

We will prove $(\operatorname{Du} 1+(\mathrm{d}))$ and $(5+(\mathrm{d}))$ as examples. The remaining cases can be shown in a similar way. Let us take $A \in$ For $_{\mathbf{C M F}}$.

(Du1+(d)) We assume $\models_{\mathrm{C}} \square A \rightarrow \neg \downarrow \neg A$, then $\forall_{\mathfrak{M} \in \mathrm{C}} \forall_{w \in W \mathfrak{M}} \mathfrak{M}, w \models$ $\square A \rightarrow \neg \diamond \neg A$, and $\square A \mathrm{R}_{w} \neg \diamond \neg A$. Therefore, by (d), $\mathrm{d}(A) \mathrm{R}_{w} \mathrm{~d}(\neg \neg A)$. On the other hand, suppose $\forall_{\mathfrak{M} \in \mathrm{C}} \forall_{w \in W^{\mathfrak{M}}} \mathrm{d}(A) \mathrm{R}_{w} \mathrm{~d}(\neg \neg A)$. Given a model $\mathfrak{M} \in \mathrm{C}$ and any $w \in W^{\mathfrak{M}}$, obviously, as in the classical modal logics $\mathfrak{M}, w \not \models \square A$ or $\mathfrak{M}, w \models \neg \diamond \neg A$. As $\mathrm{d}(A) \mathrm{R}_{w} \mathrm{~d}(\neg \neg A)$, from (d) we have $\square A \mathrm{R}_{w} \neg \oslash \neg A$, which shows that $\mathfrak{M}, w \models \square A \rightarrow \neg \vee \neg A$. Hence $\models_{\mathrm{C}} \square A \rightarrow$ $\neg \diamond \neg A$.

$(5+(\mathrm{d}))$ Assume $\mathrm{C}$ is based on the Euclidean frames. Suppose $\models_{\mathrm{C}}$

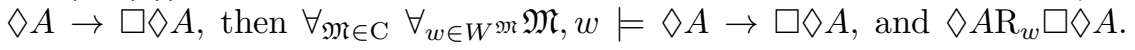


Therefore, by (d), $\mathrm{d}(A) \mathrm{R}_{w} \mathrm{~d}(A)$. On the other hand, suppose $\forall_{\mathfrak{M} \in \mathrm{C}} \forall_{w \in W^{\mathfrak{M}}}$ $\mathrm{d}(A) \mathrm{R}_{w} \mathrm{~d}(A)$. Given model $\mathfrak{M}$ and any $w \in W^{\mathfrak{M}}$. As $Q^{\mathfrak{M}}$ is Euclidean then as in the classical modal logics $\mathfrak{M}, w \not \models \diamond A$ or $\mathfrak{M}, w \models \square \diamond A$. As $A \mathrm{R}_{w} A$, from (d) we have $\diamond A \mathrm{R}_{w} \square \diamond A$, which shows that $\mathfrak{M}, w \models \diamond A \rightarrow \square \diamond A$. Hence $\models_{\mathrm{C}} \diamond A \rightarrow \square \diamond A$.

The above claim introduces different conditions imposed on relation $\mathrm{R}$ than the former ones. They are located on the right-side of the parts of the claim. We would like to point out some interesting things. It seems that a further refinement could be imposing on models the constraint of reflexivity of relation $\mathrm{R}$ in all worlds, since most of the cases state reflexivity for in $\mathrm{d}\left(\right.$ For $\left._{\mathbf{C M F}}\right)=$ For $_{\mathbf{C F}}$ as a sufficient and necessary condition. But most is not all. In three cases we have exceptions.

First, axiom $\mathrm{K}$ does not imply general reflexivity $A \mathrm{R}_{w} A$, but its special instance $(A \rightarrow B) \mathrm{R}_{w}(A \rightarrow B)$. It shows its extraordinary status in the modal logic.

Second, both forms of dual Du1, Du2 are equivalent with almost reflexivity condition: $A \mathrm{R}_{w} \neg \neg A, \neg \neg A \mathrm{R}_{w} A$. Although formulas: $A$ and $\neg \neg A$ are different from the syntactic point of view, let us note that we operate with the classical, Boolean negation $\neg$. So it looks reasonable to add as the next constraint:

$$
(\neg \neg) \quad A \mathrm{R}_{w} B \Longleftrightarrow \neg \neg A \mathrm{R}_{w} B \Longleftrightarrow A \mathrm{R}_{w} \neg \neg B .
$$

It says that double (classical!) negation has no influence on being connected. Condition $(\neg \neg)$ imposed on the models could be the next enhancement of our modal Boolean connexive logics, of course.

Finally, we may take connexive models $\mathfrak{M}=\left\langle W, Q,\left\{\mathrm{R}_{w}\right\}_{w \in W}, v\right\rangle$ defined by (Ma1), (Ma2), (Mb1), (Mb2) (or additionally by (Mc1)) and by one of the weaker conditions:

$$
A \mathrm{R}_{w} B \Rightarrow \mathrm{d}(A) \mathrm{R}_{w} \mathrm{~d}(B) \quad \text { for all } w \in W^{\mathfrak{M}}
$$

Both, (d1) and (d2), put together are equivalent to condition (d). Separately, they make equivalences in claim 6.1 invalid, reducing it to the 
suitable implications. The logics we obtain by exactly one of conditions (d1) or (d2) are probably stronger then the logics without the demodalization function, but weaker than the logics determined by models satisfying condition (d). The issue needs a further examination.

By CONST (constraints) we will denote the set of all conditions (without (d1) and (d2), separately) imposed on models $\left\langle W, Q,\left\{\mathrm{R}_{w}\right\}_{w \in W}, v\right\rangle$ we have introduced so far. Hence, CONST consists of (Ma1), (Ma2), (Mb1), (Mb2), (Mc1), (K), (Du1), (Du2), (D), (T), (4), (B), (5), (d), (K+(d)), $(\operatorname{Du} 1+(\mathrm{d})),(\mathrm{Du} 2+(\mathrm{d})),(\mathrm{D}+(\mathrm{d})),(\mathrm{T}+(\mathrm{d})),(4+(\mathrm{d})),(B+(\mathrm{d})),(5+(\mathrm{d}))$.

\section{Tableaux for the modal Boolean connexive logics}

Now, we shall outline the tableau approach to our logics in a similar way we do it in [6]. Also similarly, we will be governed here by a strategy adopted in paper [3] which introduced a formalized tableau theory from some modal logics. Let us, however, disregard the formal concepts in favour of stressing the crucial points which determine the completeness of the tableau approach related to the semantically designated consequence relations.

To this end, we shall need a new language - a language of tableau proofs. We assume as a set of expressions Ex union of four sets. They are in turn:

- $\{\operatorname{irj}: i, j \in \mathbb{N}\}$

- $\left\{\langle A, i\rangle: A \in\right.$ For $\left._{\mathrm{CMF}}, i \in \mathbb{N}\right\}$

- $\left\{\langle A R B, i\rangle: A, B \in\right.$ For $\left._{\mathbf{C M F}}, i \in \mathbb{N}\right\}$

- $\left\{\langle A \not R B, i\rangle: A, B \in\right.$ For $\left._{\mathbf{C M F}}, i \in \mathbb{N}\right\}$.

We use notation $R$ instead of $\mathrm{R}$ on purpose, to differentiate the tableau language notation $R$ from the relation in model $\mathrm{R}$. Intuitively, $r$ is a tableau counterpart of the accessibility relation, so for the world denoted by $i$ there is an accessible world denoted by $j$. Next, $\langle A, i\rangle$ means that formula $A$ is true at possible world $i,\langle A R B, i\rangle$ means that the relating relation holds between $A$ and $B$ at world $i$, while $\langle A R B, i\rangle$ that it does not.

Now, all tableau proofs are carried out in language Ex. Usually, we remove external, square brackets, so instead of $\langle A, i\rangle,\langle A R B, i\rangle,\langle A R B, i\rangle$, we just write: $A, i ; A R B, i ; A R B, i$, respectively.

As a tableau inconsistent set of expressions (that closes given branch) we treat one comprising at least one of the pairs: $A, i$ and $\neg A, i$ or $A R B, i$ and $A R B, i$, for some $A, B \in$ For $_{\mathbf{C M F}}$ and $i \in \mathbb{N}$. Clearly, a set is a tableau 
consistent set of expressions iff it is not a tableau inconsistent set of expressions.

Let us go to the tableau rules. For the formulas with the main Boolean connectives: $\neg, \wedge, \vee$, we shall assume the standard tableau rules in the modal context, so with label $i \in \mathbb{N}$ :

$$
\begin{aligned}
& (\wedge) \frac{A \wedge B, i}{A, i ; B, i} \quad(\neg \wedge) \frac{\neg(A \wedge B), i}{\neg A, i \mid \neg B, i} \\
& \text { ( }) \frac{A \vee B, i}{A, i \mid B, i} \quad(\neg \vee) \frac{\neg(A \vee B), i}{\neg A, i ; \neg B, i} \quad(\neg \neg) \frac{\neg \neg A, i}{A, i}
\end{aligned}
$$

Let us remind that the formulas do not include ones with a material implication. For the relating implication, as the main connective, we assume the tableau rules introduced in [4] also modified to the modal context. So, let $i \in \mathbb{N}$ :

$$
(\rightarrow) \frac{A \rightarrow B, i}{A R B, i ; \neg A, i \mid A R B, i ; B, i} \quad(\neg \rightarrow) \quad \frac{\neg(A \rightarrow B), i}{A, i ; \neg B, i \mid A \not R B, i} .
$$

Next, we add standard tableau rules for $\diamond, \square$ and their interactions with $\neg$.

$$
\text { (口) } \frac{\square A, i ; i r j}{A, j} \quad(\neg \square) \frac{\neg \square A, i}{\diamond \neg A, i} \quad(\diamond) \frac{\diamond A, i}{i r j ; A, j} \quad(\neg \diamond) \frac{\neg \nabla A, i}{\neg \square A, i}
$$

where $i, j \in \mathbb{N}$ and in the case of tableau rule $(\diamond)$ index $j$ does not appear on the branch.

The rules we have considered so far form the base for any modal Boolean connexive logic, we call the set of them BTR (basic tableau rules).

Now we add also well known rules for some extensions of the normal modal logics. They will be mixed with specific rules for connexive properties that are given in the further part. We assume tableau rules for the following properties of relation of accessibility $Q$ in a model, respectively for: seriality, reflexivity, transitivity, symmetry, and Euclidean property: 


$$
\begin{array}{lll}
(\text { ser }) \overline{i r j} & (\text { ref }) \overline{i r i} & (\text { tran }) \frac{i r j ; j r k}{i r k} \\
(\text { symm }) \frac{i r j}{j r i} & (\text { eucl }) \frac{i r j ; i r k}{j r k} &
\end{array}
$$

for all $i, j, k \in \mathbb{N}$. Surely, in the case of (ser) $i$ appeared on the branch, where $j$ is new; in the case of (ref) $i$ just appeared on the branch.

The next rules will be given for the specific conditions we introduced. First we reformulate to the modal context tableau rules for the Aristotelian and Boethian conditions given in [6]. Hence rules (Ra1), (Ra2), (Rb1), (Rb1'), (Rb2), (Rb2'), (Rc1) proposed in [6] are remade by adding indexes to the expressions. Later, in the presentation of all succeeding rules we always assume that $i \in \mathbb{N}$.

For the logics defined by conditions (Ma1), (Ma2) we have rules:

$$
\text { (RMa1) } \frac{A R \neg A, i}{A \not R \neg A, i} \quad(\mathrm{RMa} 2) \frac{\neg A R A, i}{\neg A \not R A, i}
$$

For the logics defined by condition (Mb1) we have two tableau rules:

$$
\begin{array}{ll}
\text { (RMb1) } & \frac{A R B, i}{A \not R \neg B, i} \\
\left(\mathrm{RMb1}^{\prime}\right) & \frac{(A \rightarrow B) R \neg(A \rightarrow \neg B), i}{(A \rightarrow B) R \neg(A \rightarrow \neg B), i}
\end{array}
$$

For the logics defined by condition (Mb2) we also have two rules that work together as well:

$$
\begin{aligned}
& (\mathrm{RMb} 2) \frac{A R \neg B, i}{A \not R B, i} \\
& \left(\mathrm{RMb}^{\prime}\right) \frac{(A \rightarrow \neg B) \not R \neg(A \rightarrow B), i}{(A \rightarrow \neg B) R \neg(A \rightarrow B), i}
\end{aligned}
$$

In fact, both (RMb1') and (RMb2') work in a similar way, since conditions (Mb1) and (Mb2) feature a common property: if $A \mathrm{R}_{w} B$, then 
$A \widetilde{\mathrm{R}}_{w} \neg B$. Hence, when dealing with a logic defined in this paper by conditions (Mb1) and (Mb2) we only adopt one rule. And finally, we also have a rule for the logic defined by condition (Mc1):

$$
\text { (RMc1) } \frac{\neg A R \neg B, i}{A \not R B, i} .
$$

Below, we have tableau rules for the suitable semantic conditions of the modal axioms that are related to claim 5.2 and claim 5.3, before the demodalization strategy.

For $(\mathrm{K})$ we have two tableau rules:

$$
\text { (RK1) } \frac{\square(A \rightarrow B) \not R \square A \rightarrow \square B, i}{\square(A \rightarrow B) \mathrm{R} \square A \rightarrow \square B, i} \quad \text { (RK2) } \frac{\square A \not R \square B, i}{\text { irj;ARRB,j }}
$$

In the case of (RK2) label $j$ must be new.

For (Du1), (Du2), we have only one tableau rule for each:

$$
\text { (RDu1) } \frac{\square A R \neg \checkmark \checkmark \neg A, i}{\square A R \neg \vee \neg A, i} \quad \text { (RDu2) } \quad \frac{\neg \oslash \neg A \not R \square A, i}{\neg \diamond \neg A R \square A, i}
$$

For (D), (T), (4), (B), (5) we have two tableau rules for each: one for the relating relation, one for accessibility $Q$, in turn:

$$
\begin{aligned}
& \text { (RD) } \frac{\square A R \diamond A, i}{\square A R \diamond A, i} \text { (ser) } \\
& \text { (RT) } \frac{\square A R A, i}{\square A R A, i} \quad \text { (ref) } \\
& \text { (R4) } \frac{\square \square A R A, i}{\square \square A R \square A, i} \text { (tran) } \\
& \text { (RB) } \frac{A R \square \diamond A, i}{A R \square \diamond A, i} \quad(\text { symm }) \\
& \text { (R5) } \frac{\diamond A R \square \diamond A, i}{\diamond A R \square \diamond A, i} \quad(\text { eucl })
\end{aligned}
$$


After assuming the demodalization property (condition (d)) (claim 5.3), we must add different tableau rules. The demodalization itself requires:

$$
(\mathrm{Rd} \Rightarrow) \frac{A R B, i}{\mathrm{~d}(A) R \mathrm{~d}(B), i} \quad(\mathrm{Rd} \Leftarrow) \quad \frac{A \not R B, i}{\mathrm{~d}(A) \not R \mathrm{~d}(B), i}
$$

For conditions $(\mathrm{K}+(\mathrm{d})),(\mathrm{Du} 1+(\mathrm{d})),(\mathrm{Du} 2+(\mathrm{d}))$, we additionally have:

$$
\begin{array}{ll}
\text { (RKd) } \frac{(A \rightarrow B) R R(A \rightarrow B), i}{(A \rightarrow B) R(A \rightarrow B), i} & \\
\text { (RDu1d) } \frac{A \not R \neg \neg A, i}{A R \neg \neg A, i} & \text { (RDu2d) } \frac{\neg \neg A \not R A, i}{\neg \neg A R A, i}
\end{array}
$$

For the remaining conditions we assume reflexivity of $\mathrm{R}_{w}$, for all worlds in the model, so we have a specific tableau rule:

$$
\text { (RrefR) } \frac{A R A, i}{A R A, i}
$$

Finally, we can formulate the tableau rules for $(D+(d)),(T+(d))$, $(4+(d)),(B+(d)),(5+(d))$, so D, T, 4, B, 5 under demodalization (d). They are combined with (RrefR) and a suitable condition on accessibility and relating $\mathrm{R}$ :

$$
\begin{aligned}
& (\mathrm{D}+(\mathrm{d})):(\text { ser }),(\text { RrefR }) \\
& (\mathrm{T}+(\mathrm{d})):(\text { ref }),(\text { RrefR }) \\
& (4+(\mathrm{d})):(\text { tran }),(\text { RrefR }) \\
& (\mathrm{B}+(\mathrm{d})):(\text { symm }),(\text { RrefR }) \\
& (5+(\mathrm{d})):(\text { eucl }),(\text { RrefR }) .
\end{aligned}
$$

For simplification, let us call the expressions in the tableau rule numerator input, while those in denominator output. Some rules, e.g. $(\rightarrow),(\neg \rightarrow)$ and those for the Boolean connectives may have more than one output. 
Now let set TRCONST (tableau rules for constraints) contain tableau rules introduced for particular conditions: (ser), (ref), (tran), (symm), (eucl), (RMa1), (RMa2), (RMb1), (RMb1'), (RMb2), (RMb2'), (RMc1), (RK1), (RK2), (RDu1), (RDu2), (RD), (RT), (R4), (RB), (R5), (Rd $\Rightarrow$ ), $(\mathrm{Rd} \Leftarrow),(\mathrm{RKd}),(\mathrm{RDu} 1 \mathrm{~d}),(\mathrm{RDu} 2 \mathrm{~d})$, (RrefR).

Let us now introduce a concept which is important for the tableau issues, which in a certain sense is an extension of the concept of truthness in model from the formulas on all expressions from Ex.

Definition 7.1. [Set of indexes] By function Ind: $\{X: X \subseteq \mathrm{Ex}\} \longrightarrow \mathrm{P}(\mathbb{N})$ we mean a mapping for all $i, j \in \mathbb{N}$ and for all $X \subseteq$ Ex satisfying conditions:

- if $X=\{i r j\}$, then $\operatorname{Ind}(X)=\{i, j\}$,

- for all $A, B \in$ For $_{\mathrm{CMF}}$ :

* if $X=\{\langle A, i\rangle\}$, then $\operatorname{Ind}(X)=\{i\}$,

$*$ if $X=\{\langle A R B, i\rangle\}$, then $\operatorname{Ind}(X)=\{i\}$,

$*$ if $X=\{\langle A \not R B, i\rangle\}$, then $\operatorname{Ind}(X)=\{i\}$,

- $\operatorname{Ind}(X)=\bigcup\{\operatorname{lnd}(\{y\}): y \in X\}$.

Function Ind collects indexes contained in expressions from a given subset of Ex.

Definition 7.2 (Model suitable to the set of expressions). Let $\mathfrak{M}=$ $\left\langle W, Q,\left\{\mathrm{R}_{w}\right\}_{w \in W}, v\right\rangle$ be a model and $X \subseteq$ Ex. Model $\mathfrak{M}$ is suitable to $X$ iff there exists a function $g$ from the set of indexes contained in expressions from $X$ to $W$, i.e. $g: \operatorname{lnd}(X) \longrightarrow W$, such that, for any $A \in$ For $_{\text {CMF }}$ and $i, j \in \mathbb{N}$ :

- if $\operatorname{ir} j \in X$, then $Q(g(i), g(j))$

- if $\langle A R B, i\rangle \in X$, then $A \mathrm{R}_{g(i)} B$

- if $\langle A R B, i\rangle \in X$, then $A \widetilde{\mathrm{R}}_{g(i)} B$

- if $\langle A, i\rangle \in X$, then $\mathfrak{M}, g(i) \vDash A$.

Making use of the provided concept of suitable model and conducting the inspection of the provided tableau rules, we are able to demonstrate that if model $\left\langle W, Q,\left\{\mathrm{R}_{w}\right\}_{w \in W}, v\right\rangle$ of given type, fulfilling some of the conditions in CONST, is suitable to set of expressions $X \subseteq$ Ex, then application of a selected tableau rule relevant to the conditions extends set $X$ to add expressions for which $\left\langle W, Q,\left\{\mathrm{R}_{w}\right\}_{w \in W}, v\right\rangle$ is still suitable. 
For convenience with formulation of the further theorems, let us introduce certain function. Now we define: $f:$ CONST $\longrightarrow\{Z: Z \subseteq$ TRCONST $\}$ which to each condition assigns the corresponding tableau rules, thus:

$$
\begin{gathered}
f((\mathrm{Ma} 1))=\{(\mathrm{RMa} 1)\} \\
f((\mathrm{Ma} 2))=\{(\mathrm{RMa} 2)\} \\
f((\mathrm{Mb} 1))=\left\{(\mathrm{RMb} 1),\left(\mathrm{RMb} 1^{\prime}\right)\right\} \\
f((\mathrm{Mb} 2))=\left\{(\mathrm{RMb}),\left(\mathrm{RMb} 2^{\prime}\right)\right\} \\
f((\mathrm{Mc} 1))=\{(\mathrm{RMc} 1)\} \\
f((\mathrm{~K}))=\{(\mathrm{RK} 1),(\mathrm{RK} 2)\} \\
f((\mathrm{Du} 1))=\{(\mathrm{RDu} 1)\} \\
f((\mathrm{Du} 2))=\{(\mathrm{RDu} 2)\} \\
f((\mathrm{D}))=\{(\mathrm{RD}),(\text { ser })\} \\
f((\mathrm{~T}))=\{(\mathrm{RT}),(\text { ref })\} \\
f((4))=\{(\mathrm{R} 4),(\text { tran })\} \\
f((\mathrm{~B}))=\{(\mathrm{RB}),(\text { symm })\} \\
f((5))=\{(\mathrm{R} 5),(\text { eucl })\} \\
f((\mathrm{~d}))=\{(\mathrm{Rd} \Rightarrow),(\mathrm{Rd} \Leftarrow)\} \\
f((\mathrm{~K}+(\mathrm{d})))=\{(\mathrm{Rd} \Rightarrow),(\mathrm{Rd} \Leftarrow),(\mathrm{RKd})\} \\
f((\mathrm{Du} 1+(\mathrm{d})))=\{(\mathrm{Rd} \Rightarrow),(\mathrm{Rd} \Leftarrow),(\mathrm{RDu} 1 \mathrm{~d})\} \\
f((\mathrm{Du} 2+(\mathrm{d})))=\{(\mathrm{Rd} \Rightarrow),(\mathrm{Rd} \Leftarrow),(\mathrm{RDu} 2 \mathrm{~d})\} \\
f((\mathrm{D}+(\mathrm{d})))=\{(\mathrm{Rd} \Rightarrow),(\mathrm{Rd} \Leftarrow),(\mathrm{RrefR}),(\text { ser })\} \\
f((\mathrm{~T}+(\mathrm{d})))=\{(\mathrm{Rd} \Rightarrow),(\mathrm{Rd} \Leftarrow),(\mathrm{RrefR}),(\text { ref })\} \\
f((4+(\mathrm{d})))=\{(\mathrm{Rd} \Rightarrow),(\mathrm{Rd} \Leftarrow),(\mathrm{RrefR}),(\text { tran })\} \\
f((\mathrm{~B}+(\mathrm{d})))=\{(\mathrm{Rd} \Rightarrow),(\mathrm{Rd} \Leftarrow),(\mathrm{RrefR}),(\text { symm })\} \\
f((5+(\mathrm{d})))=\{(\mathrm{Rd} \Rightarrow),(\mathrm{Rd} \Leftarrow),(\text { RrefR }),(\text { eucl })\} .
\end{gathered}
$$

Let us now phrase a proposition.

Claim 7.1 (Rules sound to model). Let $X \subseteq$ Ex and $U \subseteq$ CONST. Let $\left\langle W, Q,\left\{R_{w}\right\}_{w \in W}, v\right\rangle$ be a model for For ${ }_{C M F}$ defined by set of conditions $U$. Let $\left\langle W, Q,\left\{R_{w}\right\}_{w \in W}, v\right\rangle$ be suitable to $X$. If some of the tableau rules that belong to:

1. BTR

2. $\bigcup f(U)$

were applied to set $X$, then $\left\langle W, Q,\left\{R_{w}\right\}_{w \in W}, v\right\rangle$ is suitable at least to one output obtained through the application of this rule. 
Proof: Let $X \subseteq$ Ex and model $\mathfrak{M}=\left\langle W, Q,\left\{\mathrm{R}_{w}\right\}_{w \in W}, v\right\rangle$, for some $U \subseteq$ CONST, satisfy the above assumptions. If $\mathfrak{M}$ is suitable to $X$, then there exists function $g: \operatorname{lnd}(X) \longrightarrow W$ that satisfies conditions from definition 7.2.

The thesis for the rules from BTR follows from the definition of truthness for For ${ }_{\mathrm{CMF}}$, thus the proposition thesis occurs for 1, and is examined several times (see for example [3]), [4]).

The claim also holds for specific rules from TRCONST. Most of them are negative. It means that if we use one of them we immediately obtain a tableau inconsistent set. So they are inapplicable in these instances as they would contradict the assumption. For instance, if $X$ comprised expression $\langle A R \neg A, i\rangle$, then the model could not be suitable for $X$, if it meets condition (Ma1) and rule:

$$
\text { (RMa1) } \frac{A R \neg A, i}{A R \neg A, i}
$$

would introduce the tableau inconsistency to the proof.

Only rules (RMb1), (RMb2), (RMc1), (RK2), (Rd $\Rightarrow),(\mathrm{Rd} \Leftarrow)$ (taking into account set TRCONST) are positive. Thus they do not introduce the tableau inconsistency directly. The non-modal counterparts of (RMb1), (RMb2), (RMc1) we examined in [6]. Let us consider (RK2) and for example $(\mathrm{Rd} \Rightarrow)$ (checking of the other direction $(\mathrm{Rd} \Leftarrow)$ is similar).

Tableau rule:

$$
\text { (RK2) } \frac{\square A \not R \square B, i}{\operatorname{irj;ARB,j}}
$$

where label $j$ must be new, corresponds to condition (2) $\forall_{u \in W}(w Q u \Rightarrow$ $\left.A \mathrm{R}_{u} B\right) \Rightarrow \square A \mathrm{R}_{w} \square B$, from claim 5.2.

So, let us assume that (RK2) was applied to $X$. Then, $\langle\square A R \square B, i\rangle \in$ $X$, and $\square A \widetilde{\mathrm{R}}_{g(i)} \square B$ in our model $\mathfrak{M}$. The application of the rule introduced two expressions: $i r j$ and $\langle A \not R B, j\rangle$, where $j$ is new in the proof. But, by the assumption that $\mathfrak{M}$ satisfies condition $(2) \forall_{u \in W}\left(w Q u \Rightarrow A \mathrm{R}_{u} B\right) \Rightarrow$ $\square A \mathrm{R}_{w} \square B$, there must exist such world $u \in W$ that $g(i) Q u$ and $A \widetilde{\mathrm{R}}_{u} B$. So, we extend function $g$, taking $g^{\prime}: \operatorname{lnd}(X) \cup\{j\} \longrightarrow W$, with: 


$$
g^{\prime}(k)= \begin{cases}g(k), & \text { if } k \in \operatorname{lnd}(X) \\ u, & \text { if } k=j\end{cases}
$$

Therefore, after application of (RK2) to $X$ we obtain a set to which model $\mathfrak{M}$ is still suitable, because $g^{\prime}(i) Q g^{\prime}(j)$ and $A \widetilde{\mathrm{R}}_{g^{\prime}(j)} B$.

Now we will consider one of the rules for the demodalization:

$$
(\mathrm{Rd} \Rightarrow) \frac{A R B, i}{\mathrm{~d}(A) R \mathrm{~d}(B), i}
$$

The rule corresponds to the "from-left-to-right implication" in the condition from claim 6.1:

$$
\forall_{w \in W^{\mathfrak{M}}}\left(A \mathrm{R}_{w} B \Longleftrightarrow \mathrm{d}(A) \mathrm{R}_{w} \mathrm{~d}(B)\right) .
$$

Let us assume that our model $\mathfrak{M}$ satisfies the condition. At the same time $(\mathrm{Rd} \Rightarrow)$ was applied to set $X$. It means that $\langle A R B, i\rangle \in X$, and after the application there appeared $\langle\mathrm{d}(A) R \mathrm{~d}(B), i\rangle$. However, since $A \mathrm{R}_{g(i)} B$ in the model, so by condition $(\mathrm{d}), \mathrm{d}(A) \mathrm{R}_{g(i)} \mathrm{d}(B)$, too. Hence the model is suitable to $X \cup\{\langle\mathrm{d}(A) R \mathrm{~d}(B), i\rangle\}$.

The proof of completeness of our tableau methods in relation to the presented semantics still requires a converse proposition in a sense. Let us introduce the concept of model produced by set of expressions.

Definition 7.3 (Model generated by branch). Let $X \subseteq$ Ex. Model $\langle W, Q$, $\left.\left\{\mathrm{R}_{w}\right\}_{w \in W}, v\right\rangle$ is generated by $X$ iff

- $W=\operatorname{lnd}(X)$

- $i Q j$ iff $i r j \in X$, for all $i, j \in W$

- $A \mathrm{R}_{i} B$ iff $\langle A R B, i\rangle \in X$, for all $A, B \in$ For $_{\mathbf{C M F}}, i \in W$

- $v(i, x)=1$ iff $\langle x, i\rangle \in X$, for all $x \in \operatorname{Var}, i \in W$.

Assume we have a set of tableau rules that comprises:

\section{BTR}

2. set of tableau rules $\bigcup f(U)$ specified by given set of constraints $U \subseteq$ CONST. 
If we now take a set of expressions $X \subseteq$ Ex such that:

i) it is closed under all of those rules - for all expressions from $X$ to which one of the rules is applicable, there exists one output in $X$

ii) $X$ is not a tableau inconsistent set of expressions.

then there exists a model $\left\langle W, Q,\left\{\mathrm{R}_{w}\right\}_{w \in W}, v\right\rangle$ generated by that set (the set will be called minimal closure iff it is a minimal one that satisfies i), ii); for details see [3]). It does not need to satisfy constraints $U \subseteq$ CONST, but it can be enhanced. In general, it is a model for language For $_{\mathbf{C M F}}$. Therefore, we have one more proposition.

Claim 7.2 (Model sound to rules). Let $U \subseteq$ CONST. Let $X$ be:

- a tableau consistent set of expressions

- closed under BTR $\cup \bigcup f(U)$, for some set of constraints $U$.

Then there exists a model $\left\langle W, Q,\left\{R_{w}\right\}_{w \in W}, v\right\rangle$ such that:

1. $W=\operatorname{lnd}(X)$

2. for all formulas $A \in$ For $_{C M F}$ and index $i \in W$ :

$$
\langle A, i\rangle \in X \Rightarrow\left\langle W, Q,\left\{R_{w}\right\}_{w \in W}, v\right\rangle, i \models A
$$

3. model $\left\langle W, Q,\left\{R_{w}\right\}_{w \in W}, v\right\rangle$ meets conditions $U$.

Proof: Let us make all the above assumptions. We know that set $X$ generates a model. Let $\mathfrak{M}=\left\langle W, Q,\left\{\mathrm{R}_{w}\right\}_{w \in W}, v\right\rangle$ be a model generated by $X$. Surely, $W=\operatorname{lnd}(X)$, by definition of generated model.

Now the second point:

$$
\langle A, i\rangle \in X \Rightarrow \mathfrak{M}, i \models A,
$$

for all $A \in$ For $_{\mathrm{CMF}}, i \in W$. This part of the proof is inductive.

For variables $x \in \operatorname{Var}$ and negation of variables $\neg x$, the thesis is true by definition of generated model 7.2.

For more complex expressions through examination whether the tableau rules from set BTR $\cup \cup f(U)$ introduce expressions that are sufficient for constitution of a model. For the rules from BTR it is self-explanatory. The Boolean and modal rules were examined e.g. in [3]. (It is the same in the case of the rules for the specific properties of the accessibility relation, but it concerns point three of the thesis.) And the rules for the relating implication and its negation were examined in [4]. By virtue of the truth 
conditions for the combined formulas (section 4) they introduce elements that are sufficient for the construction of a verification model in the context of any possible world.

In turn, the majority of the remaining tableau rules are negative in nature or concern the character of relation $\mathrm{R}$ in possible worlds.

For example rules: (RMa1), (RMa2), (RMb1'), (RMb2'), (RK1), (RDu1), (RDu2), (RD), (RT), (R4), (RB), (R5), (RKd), (RDu1d), (RDu2d), (RrefR) are meant to close branches within proofs rather than to validate the verification formulas. They were not even applied to the expressions from $X$ as $X$ is not a tableau inconsistent set of expressions by the assumption.

So, only tableau rules (RMb1), (RMb2), (RMc1), (RK2), (Rd $\Rightarrow)$ $(\mathrm{Rd} \Leftarrow)$ are worth checking here. This way, we are starting the examination of the final part of claim 7.2: if $\mathfrak{M}$ meets conditions $U$.

Rules (RMb1), (RMb2), (RMc1) in non-modal versions were checked in [6], but we will have a look at their modal versions here. If rule:

$$
\text { (RMb1) } \frac{A R B, i}{A \not R \neg B, i}
$$

was applied then $A \mathrm{R}_{i} B$ and so $A \widetilde{\mathrm{R}}_{i} \neg B$, by definition of generated model 7.3, as condition (Mb1) states, since $\langle A R B, i\rangle,\langle A R \neg B, i\rangle \in X$. But it is similarly in the case of:

$$
(\mathrm{RMb} 2) \frac{A R \neg B, i}{A R B, i}
$$

If it was applied then $A \mathrm{R}_{i} \neg B$, so $A \widetilde{\mathrm{R}}_{i} B$ in $\mathfrak{M}$, by definition of generated model 7.3, as condition (Mb2) states, since $\langle A R \neg B, i\rangle,\langle A R B, i\rangle \in X$. For rule:

$$
(\mathrm{RMc} 1) \frac{\neg A \not R \neg B, i}{A \not R B, i}
$$

we proceed similarly. If (RMc1) was applied to $X$, then $\langle\neg A \not R \neg B, i\rangle$, $\langle A R B, i\rangle \in X$, by definition of generated model $7.3, \neg A \widetilde{\mathrm{R}}_{i} \neg B$ and $A \widetilde{\mathrm{R}}_{i} B$ in $\mathfrak{M}$, as condition (Mc1) states that $\left(A \mathrm{R}_{i} B \Rightarrow \neg A \mathrm{R} \neg B\right)$. 
The next rule corresponds to the second constraint in $(\mathrm{K})$ :

(RK2) $\frac{\square A R \square B, i}{\operatorname{irj} ; A R B, j}, \quad$ where $j$ is new on the branch.

Let us assume that $\langle\square A \not R \square B, i\rangle \in X$. By definition of generated model 7.3, $\square A \widetilde{\mathrm{R}}_{i} \square B$ in $\mathfrak{M}$. But since (RK2) was applied to $X$, then ir $j \in X$ and $\langle A R B B, j\rangle \in X$, where $j$ is a new index. Again from the definition of generated model 7.3, we get: $i Q j$ and $A \widetilde{\mathrm{R}}_{j} B$ in model $\mathfrak{M}$. However, after transposition this exactly states the second constraint in $(\mathrm{K}): \square A \widetilde{\mathrm{R}}_{w} \square B \Rightarrow \exists_{u \in W}\left(w Q u \& A \widetilde{\mathrm{R}}_{u} B\right)$.

Now, let us look at the rules for demodalization condition (d).

$$
(\mathrm{Rd} \Rightarrow) \frac{A R B, i}{\mathrm{~d}(A) R \mathrm{~d}(B), i} \quad(\mathrm{Rd} \Leftarrow) \quad \frac{A \not R B, i}{\mathrm{~d}(A) \not R \mathrm{~d}(B), i}
$$

Since $X$ does not include all expressions Ex (it is tableau consistent), even if it is closed under the above two rules the generated model does not satisfy condition (d), since some formulas did not appear in the closure of $X$. So if we want to have a model that satisfies (d), we must close family $\left\{\mathrm{R}_{w}\right\}_{w \in W}$ in model $\mathfrak{M}$ under (d). The same we have to do for the remaining conditions: (Mb1'), (Mb2'), both points of (K), (Du1), (Du2), (D), (T), (4), (B), (5).

Next, if a model has to satisfy ( $\mathrm{K}+(\mathrm{d}))$, we must close it under (d) and $(\mathrm{K}+(\mathrm{d}))$. The same applies to $(\mathrm{Du} 1+(\mathrm{d})),(\mathrm{Du} 2+(\mathrm{d}))$.

For the rest of conditions $(D+(d)),(T+(d)),(4+(d)),(B+(d)),(5+(d))$ we close the model under (d) and reflexivity of $\left\{\mathrm{R}_{w}\right\}_{w \in W}$, so for all $w \in W$ and all $A \in$ For $_{\mathrm{CMF}}$ we put $A \mathrm{R}_{w} A$. Surely, each of the conditions also contains a modal component of the accessibility relation $Q$. But since a proper rule $(($ ser $),($ ref $),($ tran $),($ symm $)$, or $($ eucl $))$ for it was used to $X$, so relation $Q$ in the generated model $\mathfrak{M}$ has a suitable property as it is a standard knowledge [3], because $Q$ can be fully defined by $X$.

Summing up, when we close the relations $\left\{\mathrm{R}_{w}\right\}_{w \in W}$ in model $\langle W, Q$, $\left.\left\{\mathrm{R}_{w}\right\}_{w \in W}, v\right\rangle$ under conditions from $U$, we obtain model $\mathfrak{M}^{\prime}=\langle W, Q$, $\left.\left\{\mathrm{R}_{w}\right\}_{w \in W}^{\prime}, v\right\rangle$ which meets conditions $U$. In this model, all formulas that are true at world $i$ in the model based on $\left\{\mathrm{R}_{w}\right\}_{w \in W}$ are true at $i$ as well, for all $i \in W$. 
Finally, we have the theorem on the completeness of tableaux and relating semantics for the discussed connexive models.

TheOrem 7.3 (Completeness theorem). Let $U \subseteq$ CONST. Let $\models \subseteq P\left(\right.$ For $\left._{C M F}\right) \times$ For $_{C M F}$ be the consequence relation defined by the set of all models designated by set of conditions $U$. Then for any $X \subseteq$ For $_{C M F}$, $A \in$ For $_{C M F}$ the following facts are equivalent:

1. $X \models A$

2. there exists a finite subset $Y \subseteq X$ and some $i \in \mathbb{N}$ such that each minimal closure of set $\{\langle B, i\rangle: B \in Y \cup\{\neg A\}\}$ under set of tableau rules $B T R \cup \cup f(U)$ is a tableau inconsistent set of expressions.

Proof: Let us adopt the assumptions. In the theorem proof, we make use of the prior propositions. For implication $1 \Rightarrow 2$ claim 7.2 is sufficient. In turn, for implication $2 \Rightarrow 1$ claim 7.1 is sufficient.

Acknowledgments. The research of Tomasz Jarmużek presented in the following article was financed by the National Science Centre, Poland, grant No.: UMO-2015/19/B/HS1/02478.

\section{References}

[1] R. L. Epstein, Relatedness and Implication, Philosophical Studies, Vol. 36 (1979), pp. 137-173.

[2] R. L. Epstein, The Semantic Foundations of Logic. Vol. 1: Propositional Logics, Nijhoff International Philosophy Series, 1990.

[3] T. Jarmużek, Tableau Metatheorem for Modal Logics, [in:] R. Ciuni, H. Wansing, C. Willkomennen (eds.), Recent Trends in Philosphical Logic, Trends in Logic, Springer Verlag 2013, pp. 105-128.

[4] T. Jarmużek and B. Kaczkowski, On some logic with a relation imposed on formulae: tableau system F, Bulletin of the Section of Logic, Vol. 43, No. 1/2 (2014), pp. 53-72.

[5] T. Jarmużek and M. Klonowski, On logic of strictly-deontic modalities, submitted to a review.

[6] T. Jarmużek and J. Malinowski, Boolean Connexive Logics, Semantics and tableau approach, Logic and Logical Philosophy, Vol. 28, No. 3 (2019), pp. 427-448, DOI: http://dx.doi.org/10.12775/LLP.2019.003

[7] A. Kapsner, Strong Connexivity, Thought, Vol. 1 (2012), pp. 141-145. 
[8] A. Kapsner, Humble Connexivity, Logic and Logical Philosophy, Vol. 28, No. 2 (2019), DOI: http://dx.doi.org/10.12775/LLP.2019.001

[9] S. McCall, A History of Connexivity, [in:] D. M. Gabbay et al. (eds.), Handbook of the History of Logic, Vol. 11, pp. 415-449, Logic: A History of its Central Concepts, Amsterdam: Elsevier 2012.

[10] H. Omori, Towards a bridge over two approaches in connexivelogics, Logic and Logical Philosophy, Vol. 28, No. 2 (2019), DOI: http://dx.doi.org/10.12775/LLP.2019.005

[11] D. N. Walton, Philosophical basis of relatedness logic, Philosophical Studies, Vol. 36, No. 2 (1979), pp. 115-136.

[12] H. Wansing and M. Unterhuber, Connexive conditional logic. Part 1, Logic and Logical Philosophy, Vol. 28, No. 2 (2019), DOI: http://dx.doi.org/10.12775/LLP.2018.018

Department of Logic

Nicolaus Copernicus University in Toruń

Torun, Poland

e-mail: Tomasz.Jarmuzek@umk.pl

Institute of Philosophy and Sociology

Polish Academy of Sciences

Warszawa, Poland

e-mail: Jacek.Malinowski@studialogica.org 